\title{
River \& Estuary Observation Network: Refinement of Stage Height Sensor Subsystem for Low Cost and High Reliability
}

\author{
W. D. Kirkey ${ }^{1}$, C. B. Fuller ${ }^{1}$, P. O’Brien ${ }^{1}$, P. J. Kirkey ${ }^{1}$, A. Mahmoud ${ }^{2}$, A. N. Ernest ${ }^{1,2}$, and J. Guerrero ${ }^{1}$ \\ ${ }^{1}$ Research Applied Technology, Education, and Services, Inc. (RATES), Colton, New York 13625, USA \\ ${ }^{2}$ University of Texas Rio Grande Valley, Edinburg, Texas 78539-2909, USA
}

Received 15 September 2020; revised 10 October 2020; accepted 13 October 2020; published online 31 December 2020

\begin{abstract}
A system comprised of software and on-site measurements is presented for accurately obtaining water stage data from vented or non-vented submersible pressure sensors installed at autonomous stream gauging stations. The system accounts for pressure sensor offset errors, water density, and local gravitational acceleration to produce a stage height reading which is accurate to either \pm 0.01 $\mathrm{ft}( \pm 3 \mathrm{~mm})$ or to the accuracy limit of the sensor, whichever is greater. A $2^{\text {nd }}$ order polynomial expression for determination of water density from temperature and salinity is developed and found to be sufficiently accurate for this purpose. Simulated stage measurements performed in the laboratory with a commercially produced sensor showed errors of up to $\pm 0.04 \mathrm{ft}$ in reported stage when the sensor's default conversion from pressure to depth was used; the maximum error limit was reduced to $\pm 0.02 \mathrm{ft}$ when the sensor output was instead processed using the new system. A custom-designed, low-cost, versatile submersible pressure sensor is introduced and tested under the same conditions and found to exhibit a maximum error of $\pm 0.04 \mathrm{ft}$ without any sensor calibration. These new developments, integrated into previously developed inexpensive base stations, enable accurate monitoring of stage height at remote locations with low installation and operating costs.
\end{abstract}

Keywords: depth sensor, gage height, hydrologic monitoring, hydrology, level sensor, stage height, streamflow, water surface elevat ion

\section{Introduction}

Uncertainties in hydrologic modelling arise from many sources. Among these are uncertainties due to the limited spatial and temporal resolution of available data and those due to the accuracy limits of the data itself (Fan, 2019). One source of high-quality hydrologic data are networks of stream gauging stations, such as that operated by the United States Geological Survey (USGS), in which surface water stage heights are continuously monitored and converted to stream flow via experimentally determined site-specific discharge rating curves (USGS, 2020). Each of these stations typically cost over $\$ 30,000$ to install, and entail annual operating budgets on the order of $\$ 10,000$ (Royem et al., 2012). These high costs limit the number of stations that agency budgets can support, thus limiting the spatial resolution of the data produced by the network. Further, installations with this level of expense are generally not feasible for monitoring activities which are short-term and/or low-budget. Such activities may not require all of the data typically obtained by the USGS system, and/or may require additional data that are not produced by standard USGS installations. Real-Time Hydrologic Stations (RTHS) (Islam et al.,

${ }^{*}$ Corresponding author. Tel.: 315-261-2171; fax: 888-852-7470.

E-mail address: wkirkey@ ratesresearch.org (W. D. Kirkey).

ISSN: 2663-6859 print/2663-6867 online

(C) 2020 ISEIS All rights reserved. doi:10.3808/jeil.202000045.
2016), which are the backbone of the River and Estuary Observatory Network (REON), offer a cost-effective solution for collecting continuous hydrologic and/or water-quality data. These stations are designed to be robust and reliable, yet inexpensive to install, operate, and disassemble or relocate, such that they are suitable for use in either long-term or short-term monitoring activities, provided that the sensors and associated systems implemented at the stations produce data of sufficient accuracy. In this paper we introduce the SWELL (Surface Water Elevation/Level) system, which is a combination of data processing software and measurement techniques for efficient and accurate stage height monitoring that has been implemented as a subsystem of the broader RTHS system. We also introduce the SHELL (Stilling-Housed ELevation/Level) sensor, a low-cost and versatile submersible pressure sensor which generates highquality stage height data when integrated with the SWELL system.

\section{Background}

\subsection{Overview of REON and RTHS}

The network of RTHS installations and the supporting cyberinfrastructure which together comprise REON was initially established by Clarkson University and the Beacon Institute for Rivers and Estuaries to provide real-time monitoring of New York State's Hudson River Watershed and provide a plat- 
form for the in-situ testing of new monitoring technologies. Presently, this network and its underlying technology are managed by the nonprofit Research Applied Technology, Education, and Services (RATES), and continues to be used for the development and deployment of cost-effective and high-performing technologies that provide real-time data with high temporal frequency for the benefit of those who manage and/or research critical water resources. REON continues to expand, and now includes RTHS installations throughout the Indian River watershed in Northern New York as well as at several locations within the Rio Grande Valley in Southern Texas.

Table 1. Acronyms and Symbols Used in This Paper

\begin{tabular}{|c|c|}
\hline Acronyms & \\
\hline CDSHS & $\begin{array}{l}\text { Custom-Designed Stage Height Sensor of (Islam, } \\
\text { 2016) }\end{array}$ \\
\hline NAVD 88 & North American Vertical Datum of 1988 \\
\hline LT400 & $\begin{array}{l}\text { LevelTROLL } 400 \text { (Commercially available level } \\
\text { sensor, In-Situ, Inc.) }\end{array}$ \\
\hline RATES & $\begin{array}{l}\text { Research Applied Technology, Education, and } \\
\text { Services, Inc. }\end{array}$ \\
\hline REON & River and Estuary Observatory Network \\
\hline RTHS & Real-Time Hydrologic Station \\
\hline SG & Specific Gravity \\
\hline SHELL & $\begin{array}{l}\text { Stage Height ELevation / Level (sensor } \\
\text { introduced in this paper) }\end{array}$ \\
\hline SWELL & $\begin{array}{l}\text { Surface Water ELevation / Level (system } \\
\text { introduced in this paper) }\end{array}$ \\
\hline USGS & United States Geological Survey \\
\hline \multicolumn{2}{|l|}{ Symbols } \\
\hline C & Conductivity \\
\hline$D$ & Depth of underwater pressure-sensing element \\
\hline$g$ & Local gravitational acceleration \\
\hline$H$ & Height (elevation above local reference) \\
\hline Pbaro & Pressure reported by barometer \\
\hline$P_{\text {gauge }}$ & Gauge Pressure at pressure-sensing element \\
\hline$P_{n v}$ & $\begin{array}{l}\text { Absolute Pressure reported by pressure-sensing } \\
\text { element }\end{array}$ \\
\hline poffset & Pressure offset (SWELL parameter) \\
\hline$H_{B}$ & Height of Benchmark \\
\hline$\rho_{w}$ & Density of water \\
\hline$S$ & Stage Height (Height of water surface) \\
\hline$\Delta S$ & Height of pressure-sensing element \\
\hline$S_{A}$ & Absolute Salinity \\
\hline$S_{\text {offset }}$ & Stage offset (SWELL parameter) \\
\hline$T$ & Temperature \\
\hline
\end{tabular}

The RTHS design features high modularity. Virtually any sensor, whether custom-designed or commercially purchased, can be integrated into the system. Any data stream consisting of comma-delimited lines of data which is connected to the RTHS USB bus is automatically logged by the system, provided that the first two fields contain a name and a serial number which uniquely identify the sensor providing the data. For commercially purchased sensors that cannot inherently produce data in this format, a simple program implemented on a USB development board (e.g., the pjrc.com, LLC Teensy series) wired to a serial converter that accepts the sensor's output protocol (e.g., RS232) is typically a sufficient interface. To date, we have utilized a variety of hydrologic and water-quality sensors (Kirkey et al., 2018; Kirkey, 2019) with the RTHS. This includes an assortment of submersible pressure sensors, all of which report water temperature together with a pressure measurement which can be converted to water depth, stage height, or elevation: the non-vented custom-designed "stage height" sensors (CDSHS) of (Islam et al., 2016), a YSI, Inc. EXO2 with a non-vented "depth" sensor, In-Situ, Inc. LevelTROLL 400 (LT400) non-vented and LevelTROLL 700H vented "level" sensors, and the non-vented SHELL sensor introduced in this paper.

\subsection{Water Pressure Sensors: Vented vs Non-Vented}

Water-pressure based depth/level sensors can be either vented or non-vented. Vented sensors contain an internal differential pressure sensor, one side of which is exposed to the sensor's exterior, and the other to an internal cavity which is vented to the ambient atmosphere via a vented cable. Non-vented sensors contain an internal pressure sensor, in which one side is exposed to the sensor's exterior, and the other side to a sealed cavity; typically, an "absolute" pressure sensor is used, meaning that the interior cavity is under vacuum. Because the pressure measured by a non-vented sensor is the sum of water pressure and barometric pressure, a separate measurement of barometric pressure must be used in order to determine the sensor depth from a non-vented pressure measurement. Non-vented sensors are typically less accurate than vented sensors, for two reasons. First, vented sensors do not require the use of a separate measurement of barometric pressure, and so have one less potential error source. Second, the range of an absolute pressure sensor used in a non-vented sensor must be larger than that of a differential sensor used in a vented sensor, because in the former the sensor must have a range large enough to sense the sum of the barometric and water pressures, and the specified error of a pressure sensor is typically a percentage of its full-scale span. However, the up-front cost of non-vented sensors, cables, and connectors are lower than those of comparable vented sensors. Further, non-vented sensors are simpler to deploy and service in many situations, because they can be connected and disconnected underwater, whereas with vented sensors, the sensor and cable cavities must always be kept dry.

\subsection{Monitoring Stage Height with Water Pressure Sensors}

Water pressure sensors can be deployed as part of an RTHS installation in a variety of ways; Figure 1 shows three methods that we have frequently used. If a pier, seawall, or similar structure is available for use, a stilling well (for example, a piece of 4" aluminum tube) can be affixed to the structure and used to house the sensor as shown in Figure 1a. The sensor is typically mounted to a rigid bar which slides into the stilling well, and is pinned in place at the top. Conduit containing the sensor cable is run to the RTHS. This deployment method affords ready access to the sensor under all water conditions. If no vertical structure is available, a stilling well can be run at an 
angle as shown in Figure 1b, and anchored to the riverbed. This method is suitable if the riverbed slope is such that a sufficient depth can be reached with a stilling well of practical length, and if there is minimal risk of ice formation that could displace the stilling well from its anchored position. For sites where installation of a stilling well is not practical, a mount for the sensor can be driven into (or otherwise anchored to) the riverbed, as shown in Figure 1c.

(a) Sensor in vertical stilling well

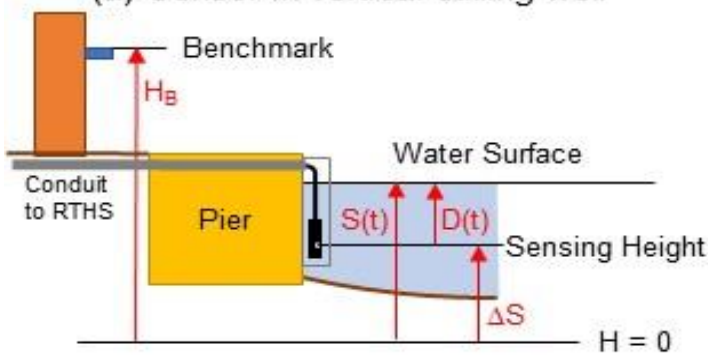

(b) Sensor in sloped stilling well

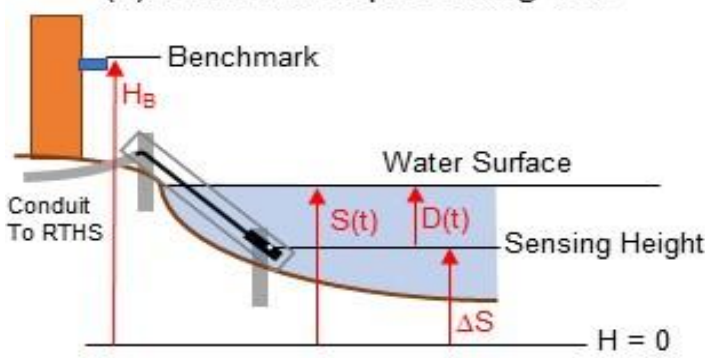

(c) Sensor affixed to mount in riverbed

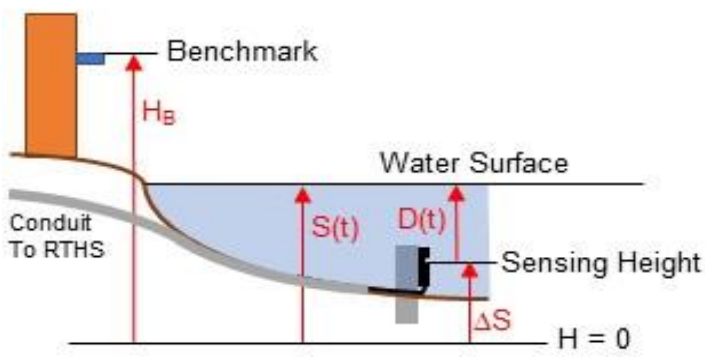

Figure 1. Three different methods for installing a depth sensor in-situ for water stage monitoring.

The elevation of the water surface will vary with time $t$. In order to report a local stage height $S(t)$ for the water surface, a reference level at which height $H=0$ must be selected; this can be selected arbitrarily, but once chosen it must remain fixed. In order to select this level, one or more benchmarks which are expected to remain at fixed elevation must be established at the site; the use of multiple benchmarks guards against the possibility an individual benchmark being compromised or destroyed. The reference height is selected by assigning a fixed benchmark height $H_{B}$ to a particular benchmark. Standard surveying equipment can be used on-site to measure the height of a local position relative to the benchmark; adding or subtracting this measurement from $H_{B}$ yields the height of said position above the $H=0$ level. If feasible, a staff gauge can be installed within the waterbody at the site at a fixed, known height above $H=0$, such that subsequent on-site water surface readings can be taken visually without the need for survey equipment.

In all cases shown in Figure 1, the gauge pressure at the sensor height, $P_{\text {gauge }}(t)$, is simply the hydrostatic pressure exerted by the water at depth $D(t)$ :

$P_{\text {gauge }}(t)=\rho_{w}(t) \times g \times D(t)$

where $g$ is the gravitational acceleration, and $\rho_{w}(t)$ is the density of the water, which can vary with time due to changes in conditions such as temperature and salinity.

As shown in Figure 1:

$S(t)=D(t)+\Delta S$

where $\Delta S$ is the height of the sensing element above the $H=0$ level.

\section{The SWELL System}

\subsection{SWELL System Design}

In the original RTHS framework, the stage reported by the system was simply the depth reported by the sensor, meaning that any replacement sensor deployed at the site had to be installed at the same depth as the original, and that any error associated with either the depth measurement or the actual depth of the sensor would be reflected in the system-reported stage data. The SWELL system is a combination of software and procedures which is designed to efficiently mitigate the impact of such errors and changes on the RTHS-reported stage height. The system is designed to sufficiently minimize any systematic errors, such that the accuracy of the reported stage values will be limited by whichever is worse: the accuracy of the gauge pressure measurement, or that of the on-site determination of the actual river stage. In the United States, survey rods and staff gauges used for the latter typically have a resolution of $0.01 \mathrm{ft}$ ( $3 \mathrm{~mm})$; this is a typical accuracy limit for direct water surface measurements (Kenney, 2010). The SWELL routines presented here aim to achieve accuracies on the order of $0.01 \%$ or lower, such that at a water depth of $10 \mathrm{ft}(3 \mathrm{~m})$, any systematic errors associated with these routines will be on the order of $0.001 \mathrm{ft}$ or less.

First, the SWELL system is designed to compensate for errors associated with using non-vented sensors. Pressure sensors typically have an inherent offset, such that they exhibit better relative accuracy than absolute accuracy. For example, the Bosch BMP085 barometers originally used in the RTHS have a specified relative accuracy of \pm 0.2 mbar and typical absolute accuracy of \pm 1.0 mbar. This is significant; $1.0 \mathrm{mbar}$ is the pressure exerted by $0.33 \mathrm{ft}(1.0 \mathrm{~cm})$ of standard surface water. For non-vented sensors, the SWELL system calculates gauge pressure at any time $t$ as follows: 
$P_{\text {gauge }}(t)=P_{n v}(t)-P_{\text {baro }}(t)+P_{\text {offset }}$

where $P_{n v}$ is the pressure reported by the non-vented water pressure sensor, $P_{\text {baro }}$ is the barometer-reported sensor, and $P_{\text {offset }}$ is a deployment-specific calibration parameter stored in the RTHS which compensates for the combined offsets of $P_{n v}$ and $P_{b a r o}$. (With vented sensors, the $P_{\text {baro }}$ value is not used.)

The SWELL system converts gauge pressure to depth $D$ and to stage $S$ as per (1) and (2), via:

$$
D(t)=\frac{P_{\text {gauge }}(t)}{g \times \rho_{w}(t)}
$$

$S(t)=D(t)+s_{\text {offset }}$

where $s_{\text {offset }}$ is a deployment-specific calibration parameter stored in the RTHS.

The value of $g$ varies with both latitude and altitude, with an overall variation on the order of $1 \%$ across the earth (Hirt et al., 2013). Thus, the SWELL system uses a site-specific value of $g$ stored in the RTHS in the calculation of (4). The density of water depends on both temperature $T$ and absolute salinity $S_{A}$, varying by about $0.5 \%$ as $T$ ranges from 0 to $40{ }^{\circ} \mathrm{C}$, and by about $3 \%$ as $S_{A}$ ranges from 0 to $35 \mathrm{~g} / \mathrm{kg}$. Modern computational tools allow the density of surface water to be calculated from $T$ and $S_{A}$ with extreme accuracy of $\pm 0.0004 \%$ (Feistel, 2008). Others have developed $6^{\text {th }}$ order polynomial expressions which mimic the results of said tools (International Oceanographic Commission et al., 2010; Roquet et al., 2015). For implementation in the SWELL system, we have developed a simplified approximation to these expressions by assuming a linear dependence of density on salinity. Figure 2 shows plots of density vs. temperature at two different salinities, calculated from the expressions in Appendix A of (Roquet et al., 2015), and bestfit second order polynomials to each plot. Assuming linearity with salinity, these yield the following expression for density:

$\rho_{w}\left(T, S_{A}\right)=f_{0}(T)+\left[f_{35}(T)-f_{0}(T)\right] \times \frac{S_{A}}{35}$

where $f_{n}(T)$ are from the fits in Figure 2 a at nominal salinity $n$ :

$$
\begin{aligned}
& f_{0}(T)=-0.005 \times\left(\frac{T}{{ }^{\circ} C}\right)^{2}+0.0235 \times \frac{T}{{ }^{\circ} C}+999.96 \\
& f_{35}(T)=-0.0043 \times\left(\frac{T}{{ }^{\circ} C}\right)^{2}-0.0854 \times \frac{T}{{ }^{\circ} C}+1028.1
\end{aligned}
$$

To test the accuracy of (6), we calculated density for all $\left\{T, S_{A}\right\}$ where $T=0,0.1,0.2, \ldots, 40^{\circ} \mathrm{C}$ and $S_{A}=0,0.1,0.2, \ldots$, $40 \mathrm{~g} / \mathrm{kg}$ using Appendix A of (Roquet et al., 2015) and using (6). The maximum difference between the two calculations was $0.013 \%$, and so (6) has accuracy on the order of $0.01 \%$ and is thus sufficient for use in the SWELL system. The real-time temperature $T(t)$ reported by the submersible pressure sensor is used in the SWELL density calculation. If a conductivity sensor is installed at the station, a real-time value $S_{A}(t)$ can be calculated from the real-time conductivity data. Otherwise, a fixed value $S_{A}$ (or a time-dependent function $S_{A}(t)$ ) can be estimated for the site and stored in the RTHS for use in SWELL calculations.

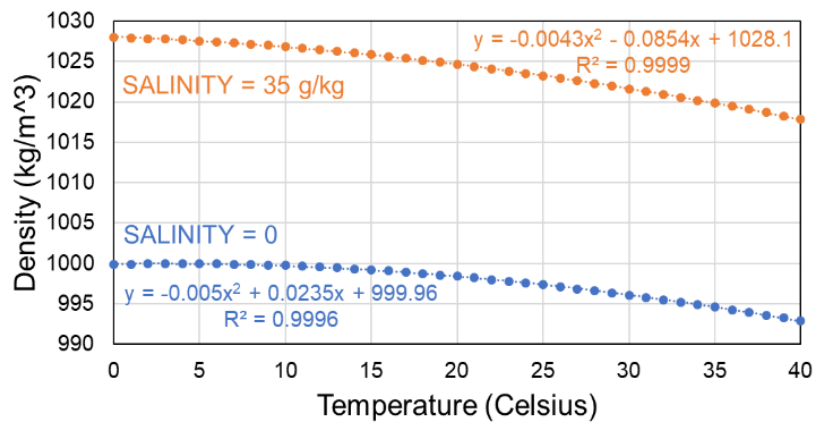

Figure 2. Water density v. temperature at salinities of 0 and $35 \mathrm{~g} / \mathrm{kg}$, calculated via (Roquet et al., 2015), and the best-fit $2^{\text {nd }}$ order polynomial for both plots.

\subsection{SWELL System Implementation}

\subsubsection{On-Site Determination of $p_{\text {offset }}$}

The $p_{\text {offset }}$ calibration value can be obtained on-site by either of two methods. In the first method, prior to installation in the water, the SHELL sensor is connected to the SWELL system and operated while in the air, such that the actual pressure at the sensor is equal to that at the barometer. $p_{\text {offset }}$ is calculated from the reported data by setting $P_{\text {gauge }}=0$ in (1). In the second method, after the system is fully installed and running, the actual depth of the water pressure sensing element is measured at a known time $t_{0}$. This value $D\left(t_{0}\right)$ is then used to together with the recorded system data to determine $p_{\text {offset }}$ via (from (1) and (2)):

$$
P_{\text {offset }}=D\left(t_{0}\right) \times g \times \rho_{w}(t)-\left[P_{n v}\left(t_{0}\right)-P_{\text {baro }}\left(t_{0}\right)\right]
$$

Relative to the second method, the first is simpler, but has the disadvantage that the submersible sensor must be kept out of the water until the rest of the system is operational, which may slow the installation process, particularly for installations such as that of Figure 1c. A drawback to the second method is that it may be difficult to accurately measure the actual depth of the sensing element; however, any error in this measurement can be largely negated in the reported stage data if the second $s_{\text {offset }}$ determination method discussed below is used. With either method, data must be managed such that only data obtained with the sensor fully installed and processed with the proper $p_{\text {offset }}$ value is ultimately reported as stage height data.

\subsubsection{On-Site Determination of $s_{\text {offse }}$}

The $s_{\text {offset }}$ value can be obtained on-site following installation by either of two methods. In the first method, DS (Figure 1 ) is measured by surveying the actual height of the water pres- 
sure sensing element, and this value is used as $s_{\text {offset }}$. A drawback to this first method is that in many installations, it may be difficult or impossible to determine the exact height of the sensing element with the desired accuracy of $\pm 0.01 \mathrm{ft}$. In the second method, the height of the water surface at a known time $t_{1}$ is measured (for example, by surveying the height of the water's edge). This value $S\left(t_{1}\right)$ is then used together with the system-reported value of $D\left(t_{1}\right)$ to calculate $s_{\text {offset }}$ via (5). Aside from being more practical, another advantage of this method over the first is that with this method, any offset in the system-reported value of $D(t)$ will be duplicated in the obtained value of $s_{\text {offset }}$, and thus eliminated in the system-reported stage height $S(t)$. With either method, once $s_{\text {offset }}$ is determined, it must be applied to all data that will ultimately be reported.

\subsubsection{Water Surface Elevation}

If the elevation of a benchmark at a site can be determined (for example, by using survey-grade GPS technology) in reference to a standard sea-level datum such as the North American Vertical Datum of 1988 (NAVD 88) (National Oceanic and Atmospheric Administration, 2020), then the water surface elevation at the site can be reported by adding the elevation of the $H=0$ level to the reported stage height. Elevation data has more "permanence" than stage height data, as stage height data has meaning only as long as the $H=0$ level remains consistent. For example, if a monitoring station which reports only stage height is removed, and a new station installed at the same location is later installed, stage data from the two installations cannot be directly compared (unless the same $H=0$ level is used in the subsequent installation is the same as in the first, which would require that a benchmark from the first installation remains known and intact).

\subsubsection{Post-Processing, Transmission, and Storage of SWELL} Data

In our default implementation, the submersible pressure sensor and the barometer each generate lines of data at a high temporal frequency (typically every 3 seconds). The RTHS software automatically generates a separate "log file" for each individual sensor; each line of these files contains a line of comma-delimited sensor data preceded by an RTHS-applied timestamp. Periodically, SWELL system software on the RTHS operates on these sensor log files to produce a "stage log file", each line of which contains: a minute-specific timestamp; the mean values for water pressure and barometric pressure obtained during each minute, each along with the associated number of measurements and standard deviation; and the $P_{\text {gauge }}$, Depth, and Stage Height values calculated from the mean pressure values. This file is zipped, transmitted to one of two central servers, and archived locally, while the original log files are archived locally but not transmitted (so as to reduce the volume of data both transmitted and archived on the servers). Normally, the stations transmit to the primary server, which then relays the data to the backup server; however, if the primary server is unreachable, the stations will relay to the data to the backup server, which subsequently relays it to the primary server. (If neither server is available, the station will periodically attempt retransmission until it successfully uploads.) On each server, data over each 5-minute block of time is averaged, and these averages are stored in a mySQL database. User interface applications can be operated on the data servers, or on separate machines which query the data servers.

The details of these data handling defaults can be customtailored to the specific needs of a particular monitoring project. For example, if data with higher temporal frequency than 1minute averages may be desired, the station can be set to also transmit the sensor-specific log files to the servers for permanent archival, such that they can be accessed in the future if desired.

\subsubsection{Reporting of SWELL Data}

By default, each RTHS station is set up with an online visualization webpage accessible from http://rths.us. Data requested via these pages is extracted from the database and placed into comma-delimited data files, which are then plotted using the open-source Dygraphs library (Dygraphs, 2020); the plots are displayed on the website, along with links for downloading the corresponding data file. Alternative interfaces for accessing the data are presently in development.

For many stations, the RTHS servers are configured to report selected results to multiple online interfaces; often, two websites are created, with one geared toward data users, and another geared toward system operators. This approach is beneficial for the SWELL system, as it produces certain types of data which will be of interest only to system operators. For example, the value of Depth reported by the SWELL system will generally be of no interest to data users (unless there are sensors for other parameters co-located with the depth sensor, in which case the sensor depth may be useful metadata for the other measured values). However, for station operations, the actual depth of the sensor is useful data for ensuring that the sensor port remains below the surface of the water. It also provides a useful parameter for troubleshooting; for example, if an error in the reported stage height is detected, the actual depth of the sensor can be checked against the reported sensor to help determine whether the error is due to the in-situ sensor or to some other component of the system. Other operationally-relevant RTHS parameters which will generally not be of interest to users include station battery voltage and charging and load currents.

\section{New SHELL Pressure Sensors}

The CDSHS originally deployed for monitoring stage height with the RTHS units are described in detail in (Paley, 2014). When deployed at RTHS stations in the vicinity of existing USGS stations, the sensors typically showed agreement to within $0.03 \sim 0.1 \mathrm{ft}(1 \sim 3 \mathrm{~cm})$ with the USGS stage height data (Islam et al., 2016). However, over extended deployments, these sensors were found to exhibit drift due to clogging of the sensor port by suspended sediments, particularly when deployed as depicted in Figure 1c. We have now custom-designed 
a new water pressure sensor, the SHELL (Stilling-Housed Elevation/Level) Sensor, shown in Figure 3, with a designed tailored for use with the SWELL system in any of the deployment configurations illustrated in Figure 1. The sensing element is embedded within two chambers, each of with is vented to the outside via small vent holes. This is intended to serve as a builtin stilling well that will inhibit suspended materials from reaching the sensing element, where it could foul the membrane and impact the accuracy of the water pressure measurement.

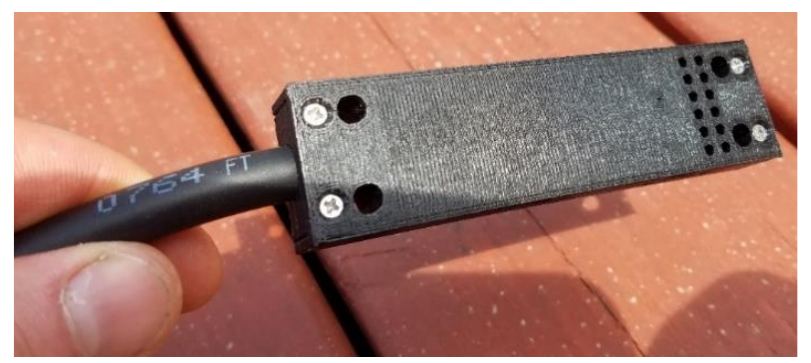

Figure 3. Photograph of SHELL sensor.

The cost of materials for the CDSHS was approximately $\$ 200$. The materials cost of the SHELL is significantly lower, totaling between $\$ 25$ and $\$ 60$ depending on the cabling configuration. Whereas the CDSHS utilized through-hole electrical components contained in a housing built from polyvinyl chloride pipe and fittings, the SHELL uses surface mount electronics and a 3D-printed polylactic acid housing, yielding a compact and versatile design. While many commercial pressure sensors are cylindrical and have a preferred deployment orientation, the SHELL's rectangular design incorporates built in mounting holes, such that it can be readily mounted with standard fasteners, and can be deployed in any orientation. A batch of four SHELL sensors requires approximately 8 hours of labor to assemble.

\section{Results and Discussion}

The LT400 and the new SHELL sensor were tested in the laboratory setup shown in Figure 4. A vertical column, open at the top, is connected to a reservoir of freshwater containing a temperature-controlled heater. Water is continuously pumped through the column, such that the water in the column remains at constant height and uniform temperature. The sensors were affixed to a weighted mount, which was lowered into the column and secured at the desired depth. Fiberglass measuring tape (not shown) attached to the mount was read at the surface of the water in order to read the depth of the mount. Changes in stage height were simulated by changing the depth of the mount by the desired amount. The accuracy this depth measurement is estimated to be $\pm 0.01 \mathrm{ft}$, which is the resolution of the measuring tape; the meniscus of the water on the measuring tape limits the ability to visually interpolate beyond this resolution. The sensors were tested at four different water temperatures. At each temperature, data was collected at simulated stage heights of $2.00,4.00,6.00$, and $8.00 \mathrm{ft}$.
The LT400 was configured to report a numeric pressure value, such that the barometric pressure correction could be applied externally. The SHELL sensor similarly reported a numeric pressure value. The RTHS system logged barometric pressure readings from a Bosch BMP180 pressure sensor. The LT400 output was converted to depth using two independent methods, Default and SWELL, where the former reflects the LT400's default internal conversion to depth. For the SHELL output, only the SWELL method was used. The Default method calculated gauge pressure by subtracting the barometer reading from the LT400-reported pressure, and then applied the conversion value of $0.70307 \mathrm{~m} / \mathrm{PSI}$ that Level TROLL series sensors use by default (In-Situ, Inc., 2015) to convert pressure to depth. The SWELL method used Equations (3), (4), and (6), with a $p_{\text {offset }}$ value obtained by Method 1 of section 4.2.1. With each method, the depth was converted to stage using (5), with a $s_{\text {offset }}$ value obtained from the output at $25.4{ }^{\circ} \mathrm{C}, 2.00 \mathrm{ft}$ stage as per Method 2 of section 4.2.2.

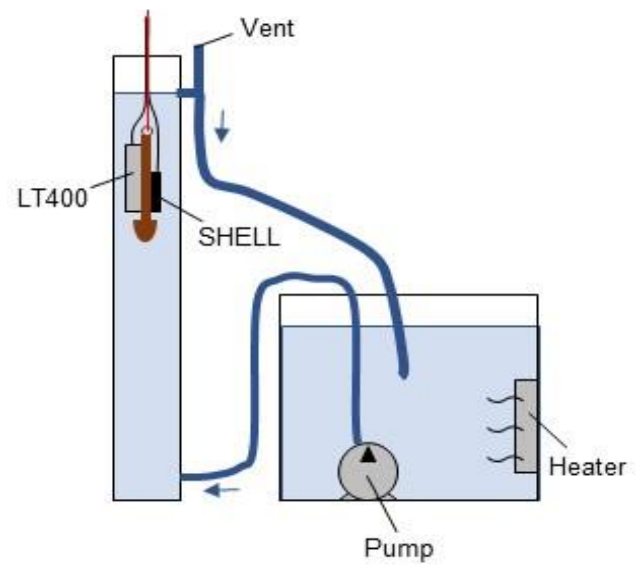

Figure 4. Diagram of laboratory setup for stage height measurements.

The reported stage values obtained with each Sensor (Method) are shown in Table 1, together with the Stage Error (Reported Stage - Actual Stage). For the LT400, both the Default and SWELL methods produce accurate output at low and moderate temperatures, with errors no larger than $0.02 \mathrm{ft}$ in magnitude (recall that the error of the actual stage measurements is $\pm 0.01 \mathrm{ft}$ ). However, at $32.5{ }^{\circ} \mathrm{C}$, the Default method yields error of $-0.04 \mathrm{ft}$, while the SWELL method remains accurate to within $\pm 0.01 \mathrm{ft}$. The LT400's internal conversion uses the coefficient $0.70307 / \mathrm{SG}$ to convert pressure in PSI to depth in meters, where SG is the specific gravity and is set to 1 by default, which pertains to water at $4{ }^{\circ} \mathrm{C}$. The actual specific gravity of water decreases with increasing temperature, which explains why the Default conversion yields an under-reported depth at elevated temperatures. These results illustrate that the details of the conversion from pressure to stage can have a significant result on the accuracy of the measurement, even after the most significant effects (such as the impact of barometric pressure on the sensor output) are accounted for.

The results obtained with the SHELL sensor are comparable to those obtained with the LT400 via the Default method, 
Table 2. Error in the Stage Reported by the LT400 and SHELL Sensors in the Laboratory When Processed with the LT400 Defaults and with the SWELL System

\begin{tabular}{|c|c|c|c|c|c|c|c|}
\hline \multicolumn{2}{|c|}{ Actual Conditions } & \multicolumn{2}{|c|}{ LT400 (Default) } & \multicolumn{2}{|c|}{ LT400 (SWELL) } & \multicolumn{2}{|c|}{ SHELL (SWELL) } \\
\hline Actual & Water & Reported & Stage Error & Reported & Stage Error & Reported & Stage Error \\
\hline Stage $(\mathrm{ft})$ & Temperature $\left({ }^{\circ} \mathrm{C}\right)$ & Stage $(\mathrm{ft})$ & $(\mathrm{ft})$ & Stage $(\mathrm{ft})$ & $(\mathrm{ft})$ & Stage $(\mathrm{ft})$ & $(\mathrm{ft})$ \\
\hline 2.00 & 11.80 & 1.985 & -0.020 & 1.981 & -0.020 & 2.026 & +0.030 \\
\hline 4.00 & 11.80 & 3.990 & -0.010 & 3.987 & -0.010 & 4.011 & +0.010 \\
\hline 6.00 & 11.80 & 5.988 & -0.010 & 5.986 & -0.010 & 6.004 & +0.000 \\
\hline 8.00 & 11.80 & 7.978 & -0.020 & 7.978 & -0.020 & 7.984 & -0.020 \\
\hline 2.00 & 17.50 & 1.995 & -0.010 & 1.992 & -0.010 & 2.010 & +0.010 \\
\hline 4.00 & 17.50 & 3.988 & -0.010 & 3.987 & -0.010 & 3.993 & -0.010 \\
\hline 6.00 & 17.50 & 5.991 & -0.010 & 5.994 & -0.010 & 5.985 & -0.020 \\
\hline 8.00 & 17.50 & 7.985 & -0.020 & 7.990 & -0.010 & 7.977 & -0.020 \\
\hline 2.00 & 24.50 & 2.000 & 0.000 & 2.000 & 0.000 & 2.000 & 0.000 \\
\hline 4.00 & 24.50 & 3.994 & -0.010 & 3.999 & -0.000 & 3.987 & -0.010 \\
\hline 6.00 & 24.50 & 5.983 & -0.020 & 5.993 & -0.010 & 5.972 & -0.030 \\
\hline 8.00 & 24.50 & 7.981 & -0.020 & 7.996 & -0.000 & 7.989 & -0.010 \\
\hline 2.00 & 32.50 & 1.991 & -0.010 & 1.995 & -0.010 & 1.994 & -0.010 \\
\hline 4.00 & 32.50 & 3.977 & -0.020 & 3.990 & -0.010 & 3.986 & -0.010 \\
\hline 6.00 & 32.50 & 5.964 & -0.040 & 5.986 & -0.010 & 5.981 & -0.020 \\
\hline 8.00 & 32.50 & 7.960 & -0.040 & 7.992 & -0.010 & 7.962 & -0.040 \\
\hline
\end{tabular}

but inferior to those obtained with the LT400 via the SWELL method. However, the SHELL sensor as presented here required no calibration, whereas the LT400 incorporates a factory calibration; the manufacturer recommends that the sensor be returned to the factory for re-calibration every $12 \sim 18$ months (In-Situ, Inc., 2019). Potential calibrations for the SHELL's pressure response will be explored in the future. Even with no calibration, the SHELL sensor shows comparable performance for stage height measurements to that of the LT400 when factory defaults are used for the conversion.

It should be noted that the LT400 includes provisions for changing settings stored in the sensor, which would allow it to mimic the SWELL conversion method and report a depth or stage; specific gravity can be changed, and values for pressure offset and level (i.e., stage) offset can be entered, but updating these parameters would require external software to make calculations and send new values to the sensor, updating for barometric pressure and specific gravity changes as needed. The SWELL system instead allows the sensor to simply be configured to report the in-water pressure (absolute pressure for nonvented sensors, gauge pressure for vented sensors), and places all of the information that depends on sources external to the sensor on the RTHS. Another model for comparison is that used by the YSI EXO2, which is a modular, multiparameter sonde that can be equipped with a vented or a non-vented depth /level sensor which can report pressure and/or depth (YSI, Inc., 2020). With a non-vented sensor, the EXO2 can be positioned in air and zeroed before use in order to correct for barometric pressure, but any subsequent changes in barometric pressure will impact the EXO2's output. Whether vented or non-vented, the EXO2 converts pressure to depth using an internal algorithm. Altitude and latitude can optionally be entered by the user to be used in the algorithm, and if the sonde is equipped with the optional $\mathrm{C} / \mathrm{T}$ sensor, the conductivity and temperature data are used as well. When used with the SWELL system, the EXO2 simply needs to report pressure. The barometric pressure is automatically used to compensate if the depth/level sensor is non-vented. Temperature and conductivity can be sourced either by the EXO2, or by some other source(s). Finally, with the SWELL system, because all site-related pa-rameters are stored on the RTHS rather than in the sensor, if a pressure sensor is replaced or relocated, none of the settings in the sensor or on the station need to be changed other than $p_{\text {offset }}$ and $s_{\text {offset }}$.

\section{Conclusions}

The SWELL subsystem for accurately and efficiently converting in-situ water pressure data into stage height or elevation data and its implementation as part of the broader RTHS system has been presented. Stage height data produced by a commercially available pressure sensor was shown to be significantly more accurate when processed using the SWELL system as opposed to using the sensor's default conversion parameters. Further, the SHELL sensor, a low-cost water pressure sensor which can be readily integrated into SWELL has been introduced. These innovations allow for accurate monitoring of surface water stage height that can be implemented cost-effectively for monitoring at remote locations.

\section{References}

Dygraphs. (2020). Dygraphs charting library. https://dygraphs.com/. Fan, Y.R. (2019). Uncertainty quantification in hydrologic predictions: A brief review. Journal of Environmental Informatics Letters, 2(2), 48-56. https://doi.org/10.3808/jeil.201900019

Feistel, R. (2008). A Gibbs function for seawater thermodynamics for -6 to $80^{\circ} \mathrm{C}$ and salinity up to $120 \mathrm{~g} \mathrm{~kg}^{-1}$. Deep Sea Research Part I: Oceanographic Research Papers, 55(12), 1639-1671. https://doi. org/10.1016/j.dsr.2008.07.004 
Hirt, C., Claessens, S., Fecher, T., Kuhn, M., Pail, R., and Rexer, M. (2013) New ultrahigh-resolution picture of Earth's gravity field. Geophysical Research Letters, 40(16), 4279-4283. https://doi.org/ $10.1002 / \mathrm{grl} .50838$

In-Situ, Inc. (2015). In-Situ modbus communication protocol version 5.10. https://in-situ.com/us/pub/media/support/documents/Modbus Manual.pdf.

In-Situ, Inc. (2019). Level TROLL 400, 500, 700, 700H Instruments Operator's Manual 0052212. https://in-situ.com/us/level-troll-400data-logger.

Intergovernmental Oceanographic Commission(IOC), Scientific Committee on Oceanographic Research (SCOR), and International Association for the Physical Sciences of the Oceans (IAPSO). (2010). The international thermodynamic equation of seawater 2010: Calculation and use of thermodynamic properties (Revised June 2015). IOC Manuals and Guides No.56, United Nations Educational, Scientific and Cultural Organization (UNESCO). http:// www.teos-10.org/pubs/TEOS-10_Manual.pdf.

Islam, M.S., Bonner, J.S., Paley, J.B., and Fuller, C.B. (2016). Lowcost stand-alone system for real-time hydrological monitoring. Environmental Engineering Science, 33(12), 929-941. https://doi. org/10.1089/ees.2016.0106

Kenney, T.A. (2010). Levels at Gaging Stations: U.S. Geological Survey Techniques and Methods, book 3, chap. A19. United States Geological Survey. https://doi.org/10.3133/tm3A19

Kirkey, W.D. (2019). New designs for submersible water quality instrumentation which achieve high in situ accuracy at low total cost.
A dissertation. Ph.D. Dissertation, School of Engineering, Clarkson University, Potsdam, N.Y., U.S.A.

Kirkey, W.D., Bonner, J.S., and Fuller, C.B. (2018). Low-cost submersible turbidity sensors using low-frequency source light modulation. IEEE Sensors Journal, 18(22), 9151-9162. https://doi.org/ 10.1109/JSEN.2018.2869368

National Oceanic and Atmospheric Administration National Geodetic Survey (NOAA National Geodetic Survey). (2020). North American Vertical Datum of 1988 (NAVD 88). https://www.ngs.noaa. gov/datums/vertical/north-american-vertical-datum-1988.Shtml.

Paley, J.B. (2014). A new low-cost real-time hydrological monitoring system: A thesis. M.S. Thesis, School of Engineering, Clarkson University, Potsdam, N.Y., U.S.A.

Roquet, F., Madec, G., McDougall, T.J., and Barker, P.M. (2015). Accurate polynomial expressions for the density and specific volume of seawater using the TEOS-10 standard. Ocean Modeling, 90, 29-43. https://doi.org/10.1016/j.ocemod.2015.04.002

Royem, A.A., Mui, C.K., Fuka, D.R., and Walter, M.T. (2012). Proposing a low-tech, affordable, accurate stream stage monitoring system. Transactions of the ASABE, 55(6), 2237-2242. https://doi. org/10.13031/2013.42512

The U.S. Geological Survey (USGS). (2020). USGS current water data for the nation. https://waterdata.usgs.gov/nwis/rt.

YSI, Inc. (2020). EXO User Manual, Revision J. https://www.ysi.com/ File\%20Library/Documents/Manuals/EXO-User-ManualWeb.pdf 